\title{
Introgression of Large Grain Size from Australian Wild Rice and Its Agronomical Importance ${ }^{+}$
}

\author{
Ryuji Ishikawa ${ }^{1, *}$, Takahiro Mishimaki ${ }^{1}$, Daiki Toyomoto ${ }^{2}$, Koki Katano ${ }^{3}$, Katsuyuki Ichitani ${ }^{2,3}$ \\ and Robert Henry ${ }^{4}$ \\ 1 Faculty of Agriculture and Life Science, Hirosaki University, Hirosaki, Aomori 036-8561, Japan; \\ h15a3030@hirosaki-u.ac.jp \\ 2 United Graduate School of Agricultural Sciences, Kagoshima University, 1-21-24 Korimoto, Kagoshima, \\ Kagoshima 890-0065, Japan; k1560237@kadai.jp (D.T.); ichitani@agri.kagoshima-u.ac.jp (K.I.) \\ 3 Faculty of Agriculture, Kagoshima University, Kagoshima 890-0065, Japan; k3145525@kadai.jp \\ 4 QAFFI, The University of Queensland, St Lucia, QLD 4073, Australia; robert.henry@uq.edu.au \\ * Correspondence: ishikawa@hirosaki-u.ac.jp \\ † Presented at the Third International Tropical Agriculture Conference (TROPAG 2019), Brisbane, Australia, \\ 11-13 November 2019.
}

Published: 7 March 2020

\begin{abstract}
There are a few wild species belonging to genus Oryza in Australia. Taxon A and Taxon $B$ have been characterized genetically and ecologically. We have backcrossed the Taxon B carrying larger seed size with Japonica type cultivated rice, Taichung 65 (T65). BC4F1 seeds selected the seed size of BC4F2 showed that Taxon B type subgroup carries 21.5\% larger seeds size than T65. The heterozygous group showed incomplete dominance in seed size. Introgressed segments suggested that the causal gene could be a single and located on chromosome 3. By using BC4F2, we characterized agronomical traits to show how the large seed size gene affects to yield. The larger seed size did not show any correlation to number of panicles and panicle length. However, it affected negative effect on number of spikelets. It is explained as Trade-off relation. The negative effect will be resolved to introduce genetic factor related to number of tillers or spikelets per panicle, or they may be tried improved ways of cultivation. The seed size is attractive to improve new varieties as "Australian native rice" to be adopted with a variety of cooking style which prefer large grain size or a variety of application of the size. Japanese Sake is another way because it generally requires larger grain size to expect white core. We are generating pure lines carrying only the gene involving to the seed size with other negative traits for cultivation.
\end{abstract}

Keywords: Oryza; grain size; introgression

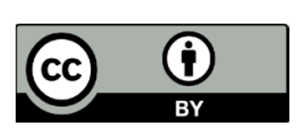

(C) 2020 by the authors. Licensee MDPI, Basel, Switzerland. This article is an open access article distributed under the terms and conditions of the Creative Commons Attribution (CC BY) license (http://creativecommons.org/licenses/by/4.0/). 